\title{
Lo sviluppo della dialisi peritoneale in Italia: esperienza di un ospedale privato accreditato
}

\author{
Silvio Bertoli, Laura Buzzi, Daniele Ciurlino \\ Unità Operativa di Medicina Generale - Sezione Nefrologia e Dialisi \\ Policlinico Multimedica, Sesto San Giovanni (Milano)
}

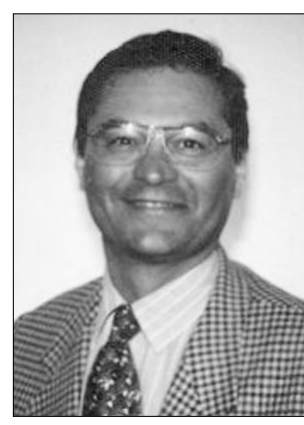

Silvio Bertoli
$\mathbf{L}_{\text {a }}$ peritoneale ambulatoriale continua (CAPD) ha subito negli ultimi anni un evidente rallentamento di crescita, non compensato da un adeguato sviluppo della dialisi peritoneale automatizzata (APD) (1).

La dialisi peritoneale (DP) è una metodica utilizzata in tutto il mondo: in Canada la prevalenza dei pazienti trattati è del $33 \%$, in America Latina del 31\%, negli Stati Uniti del 17\%, nei Paesi dell'Est del 16\%, in Europa del 14\% (2). In Italia solo il $10 \%$ dei pazienti in trattamento sostitutivo è in DP, anche se nel nostro Paese esistono diverse realtà regionali: in Lombardia il $17 \%$ dei pazienti in dialisi è in DP, mentre in alcune regioni del Sud tale percentuale diventa estremamente modesta (1). Questa eterogeneità è condizionata da due importanti aspetti: la cultura professionale del medico e il contesto socio-sanitario ed economico.

\section{Cultura professionale}

La DP, nata nel 1976, è stata divulgata negli anni Ottanta e Novanta come una metodica innovativa ed è stata successivamente confrontata con l'emodialisi (ED) (3-5). Anche se la sopravvivenza dei pazienti non è risultata significativamente differente nei due tipi di trattamento, il confronto fra DP ed ED è inficiato da un grave errore concettuale: vengono infatti raffrontate metodiche che utilizzano due membrane dialitiche diverse, una biologica e una sintetica.

In Italia è stata operata una selezione dei pazienti in DP prevalentemente negativa, considerando la DP come "scelta di ripiego" subordinata all'impossibilità di utilizzare l'ED. Da questa impostazione, di per sé sfavorevole, sono emersi soprattutto gli aspetti negativi connessi alla DP (infezioni, malnutrizione, sclerosi peritoneale), a discapito di quelli positivi rappresentati dalla riabilitazione e dalla miglior qualità della vita dei pazienti (6). È scorretto considerare le complicanze della DP come controindicazioni all'utilizzo di tale metodica; è invece compito del nefrologo conoscerne l'esistenza, per saperle curare ed evitarne l'evoluzione. Studi condotti recentemente suggeriscono il vantaggio di avvalersi di una terapia sostitutiva "integrata", in cui DP ed ED vengano utilizzate come metodiche "alla pari" seppur con le loro specificità cliniche (7).

Un'adeguata preparazione culturale nefrologica è condizione necessaria ma non sufficiente per proporre la DP a pazienti relativamente giovani: è opinione personale di non pochi medici che avere un catetere peritoneale in addome rappresenta un handicap fisico nella vita relazionale. Tale giudizio "estetico" può precludere la DP a pazienti peraltro idonei a questo trattamento.

\section{Contesto socio-sanitario ed eco- nomico}

L'invecchiamento della popolazione dialitica ha portato alla diminuzione della DP, inadatta a pazienti non autosufficienti o con partner non disponibili. Una soluzione per questi pazienti potrebbe essere un'assistenza infermieristica domiciliare, eventualmente supportata dalla partecipazione delle aziende che si occupano di DP, oppure organizzare piccoli Centri di DP in strutture protette.

Dal punto di vista economico, la DP non è una metodica costosa: il catetere peritoneale viene posizionato in regime di ricovero; il costo delle sacche di infusione è contenuto rispetto al rimborso; la metodica automatizzata, seppur più costosa della CAPD, ha un rimborso che ammortizza le spese; il costo della DP per i pazienti ricoverati è inferiore a quello del- 
l'ED; il costo delle visite ambulatoriali e degli esami di laboratorio viene rimborsato; la DP richiede un minor impiego di personale medico e infermieristico rispetto all'ED; qualora il personale infermieristico ospedaliero non fosse disponibile per addestrare i pazienti, è possibile rivolgersi alle aziende fornitrici del materiale per DP e includere nel loro service l'addestramento dei pazienti. È tuttavia consigliabile che il personale infermieristico del Centro dialisi venga addestrato alla DP, per garantire al paziente un punto di riferimento costante. Tuttavia l'impegno del medico, a nostro parere, è sicuramente più importante rispetto ai pazienti in ED, sia per problemi clinici che per problemi psico-sociali.

La nostra esperienza di lavoro nell'ambito di un ospedale privato accreditato è positiva, in quanto ci consente di realizzare un trattamento sostitutivo integrato. Parallelamente alla ED convenzionale abbiamo iniziato la DP domiciliare, preventivando alla Direzione Generale i costi e i ricavi del trattamento. Abbiamo organizzato un service per CAPD e APD, in cui sono stati inclusi la fornitura del materiale a domicilio e l'addestramento dei pazienti, risolvendo il problema dell'iniziale mancanza di personale infermieristico ospedaliero specializzato. Il service è stato stipulato con due diverse aziende, per garantirci un'alternativa nella gestione infermieristica. Abbiamo addestrato alla DP due infermiere che si occupano di ED e, contemporaneamente, ricoprono il servizio di DP dalle ore 7.00 alle ore 19.00 , per sei giorni alla settimana, con reperibilità notturna e festiva: questo permette un impiego più efficiente del personale infermieristico quando i pazienti in DP sono meno di 20; per un numero superiore, probabilmente è necessaria un'infermiera dedicata. La gestione della DP avviene tramite prestazioni ambulatoriali adeguatamente registrate; la gestione delle complicanze è in regime di ricovero.

Dopo nove mesi dall'apertura del Servizio dialisi, abbiamo 46 pazienti in trattamento sostitutivo, 38 in ED e 8 in DP, con una prevalenza di pa- zienti in DP pari al 18\%. Dobbiamo segnalare che la selezione dei nostri pazienti è stata positiva (età media 61 \pm 15 anni), con una ridotta incidenza di infezioni e malnutrizione.

La nostra esperienza in una struttura privata accreditata non ha modificato il nostro atteggiamento culturale, che nasce dall'esperienza e dagli anni di lavoro in una struttura pubblica e dal credere in una metodica che ha nella de-ospedalizzazione la sua arma vincente. Il trattamento sostitutivo domiciliare permette una riabilitazione del paziente e una qualità della vita migliore, a condizione che l'équipe medico-infermieristica creda in questa affermazione e sia in grado di motivare il paziente; la DP è tuttavia una metodica tempo-dipendente, che va sostituita quando le condizioni cliniche e/o psico-sociali lo richiedano. Riteniamo pertanto che svolgere la propria professione in un ospedale privato accreditato non rappresenti un limite al trattamento con DP: è indispensabile un corretto approccio culturale, sia clinico che economico. L'assenza di un'infermiera dedicata alla DP non ostacola lo sviluppo della metodica, in quanto aziende specializzate possono fornire, se necessarie, professionalità infermieristiche per addestrare adeguatamente i pazienti.

silvio.bertoli@multimedica.it

\section{BIBLIOGRAFIA}

1. Colasanti G, Santoro A, Salomone M, Conte F, Salvadori M, Pastorino M. Registro Italiano di Dialisi e Trapianto: Report del Registro, Anno $1998.41^{\circ}$ Congresso della SIN, Taormina, 14-17 Giugno 2000.

2. Durand PY, Kessler M, Chanliau J. Dialyse péritonéale adéquate: limites de la dialyse péritonéale continue ambulatoire (DPCA), place de la dialyse péritonéale automatisée (DPA). Nephrologie 1998; 19: 239244.

3. Maiorca R, Cancarini GC, Brunori G, et al. Comparison of long-term survival between hemodialysis and peritoneal dialysis. Adv Perit Dial 1996; 12: 79-88.

4. Fenton S, Schaubel DE, Desmueles M, et al. Hemodialysis versus peritoneal dialysis: a comparison of adjusted mortality rates. Am J Kidney Dis 1997; 30: 334-42.

5. Port FK, Wolfe RA, Bloembergen WE, Held PJ, Young EW. The study of outcomes for CAPD versus hemodialysis patients. Perit Dial Int 1996; 16: 628-33.

6. Cameron JI, Whiteside C, Katz J, Devins GM. Differences in quality of life across renal replacement therapies: a meta-comparison. Am J Kidney Dis 2000; 35: 629-37.

7. Bataille P, Coevoet B, Cuvelier D, et al. Factors determining the choice of a modality of treatment by dialysis: a study of nine dialysis centers. Nephrologie 2000; 21: 57-63. 